\title{
Uric acid, gout and the kidney
}

\author{
JS CAMERON, HA SIMMONDS
}

From the Renal Unit and Purine Laboratory, Department of Medicine, Guy's Hospital, London SEI 9RT

Uric acid is 2,6,8-trihydroxypurine, and seems to be present in almost all organisms. In snakes, reptiles, birds and spiders it is the main end-product of all nitrogenous metabolism, and these groups of animals have kidneys which are capable of secreting large amounts of uric acid. This is then excreted as a slurry of crystals, which confers the advantage that the nitrogenous end-product can be excreted with very little water, a benefit in arid environments. In contrast, the excretion of urea necessitates the loss of a relatively larger quantity of water, the size of which is dependent upon the concentrating ability of the kidney.

In mammals, uric acid is the end-product only of purine metabolism and is formed by the breakdown of purine nucleotides, which are derived from DNA and RNA, and also from other compounds important in general metabolism, such as ATP, ADP and AMP, FAD, coenzyme A and S-adenosylmethionine. In mammals, with the exception of man and closely related primates, uric acid is further broken down to allantoin by the enzyme uricase. The advantage of this is that allantoin is a very soluble compound, whereas uric acid is very insoluble.

At physiological $\mathrm{pH}$ only the hydroxyl group at the 8 position, which has a pK of $5 \cdot 4$, dissociates. Therefore, in blood and tissue fluids uric acid is overwhelmingly $(98 \%)$ in the dissociated form of $\mathrm{Ur}^{-}$, so that effectively in tissue fluids the predominant form is $\mathrm{Na}^{+} \mathrm{Ur}^{-}$, with smaller amounts of $\mathrm{K}^{+} \mathrm{Ur}^{-}$ etc. In the urine, in contrast, as the physiological range of $\mathrm{pH}$ is 4.7 to 8.0 , the proportion of $\mathrm{HUr}$ to $\mathrm{Ur}^{-}$varies greatly; in most acid urines the form is predominantly the undissociated acid. This is many times less soluble in water than sodium urate, hence the great variation in the capacity of urine of various pHs to dissolve uric acid (Fig. 1).

The toxicity of and the pathological changes caused by uric acid are entirely the consequences of its insolubility. This would not matter so much, except perhaps for a tendency to form crystals or stones in acid urine, were it not for one other peculiarity which primates also display, namely that the renal tubule reabsorbs around $90 \%$ of the filtered urate. There is great variation between different mammals in the renal handling of uric

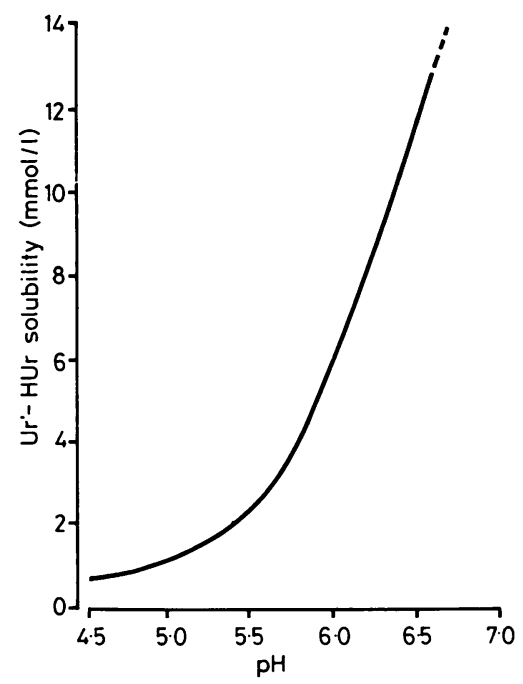

Fig. 1 The solubility of the $\mathrm{HUr} / \mathrm{H}^{+} \mathrm{Ur}^{-}$system in urine, in which the principal cation is $\mathrm{Na}^{+}$.

acid, ${ }^{1}$ from net secretion with uric acid clearances two to three times the glomerular filtration rate, as in the pig and some rabbits, down to man, who has a net reabsorption of uric acid in the renal tubule higher than any other mammal except closely related primates.

The result of the deletion of the enzyme uricase and the extensive tubular reabsorption of uric acid is that the concentration of uric acid in human body fluids is not far off the limit of solubility in plasma at $37^{\circ} \mathrm{C}$ (about $0.45 \mathrm{mmol} / \mathrm{l}$ ), especially in males, who have a higher level of plasma uric acid than children or premenopausal females. This contrasts sharply with other mammals whose plasma uric acid is, in general, less than $0.03 \mathrm{mmol} / \mathrm{l}$ : in the pig it is as low as $0.002 \mathrm{mmol} / \mathrm{l}$. The evolutionary advantage of this production and retention of uric acid is not clear, especially as it carries with it the risk of gouty disease.

\section{Handling of uric acid by the kidney}

Urate is handled by the human kidney in a complex fashion which has a number of clinically important 
consequences. ${ }^{2}$ It includes filtration, followed by a bidirectional tubular transport system involving reabsorption, secretion and finally postsecretory reabsorption (Fig. 2). The exact sites of these transport systems within the nephron are not known, and they may coexist extensively throughout the proximal tubule. It is likely that the brief account given here will have to be changed in the future.

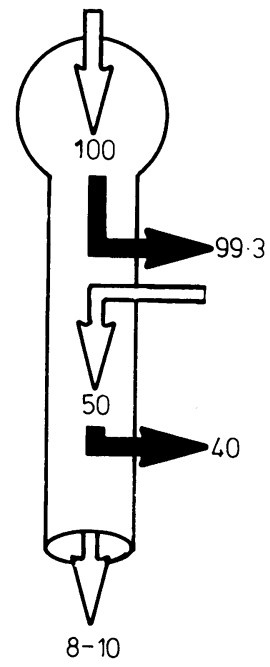

Fig. 2 Current concepts of the handling of urate by the human renal tubule. The numbers represent the amount of urate handled as a percentage of the filtered load.

Plasma proteins, principally albumin, are capable of binding uric acid, but the extent of such binding is uncertain in human plasma at $37^{\circ} \mathrm{C}$. Binding, however, appears appreciable at $4{ }^{\circ} \mathrm{C}$; this is responsible for frequent statements in the past that uric acid is extensively bound (ca $15 \%$ ), and therefore not freely available for filtration. Current evidence suggests that the contrary is true under physiological circumstances in man, and that virtually $100 \%$ of plasma urate may be filtered at the glomerulus.

Calculations suggest that some $50 \mathrm{mmol}$ of urate are filtered at the glomeruli in a normal adult every $24 \mathrm{~h}$; yet less than $4 \mathrm{mmol} /$ day are excreted after several days on a purine-free diet, and rather more on a higher purine intake. Net reabsorption is about $90 \%$ in females, and $92 \%$ in males ${ }^{3}$ this difference accounts for the higher plasma uric acid in males and is perhaps influenced by sex hormones. It is necessary to postulate only filtration and reabsorption to account for these data, but observations of uric acid clearances in excess of the glom- erular filtration rate in individuals with inherited or acquired renal tubular defects as well as in healthy individuals during urate loading coupled with probenecid treatment (a uricosuric agent), made it necessary to postulate tubular secretion as well.

At one time it was assumed that almost all the filtered urate was reabsorbed, and that urinary urate arose almost entirely from a postreabsorptive secretory mechanism. The principal evidence for this suggestion came from studies using the antituberculous agent pyrazinamide (PZA) which, in its active form pyrazinoic acid, almost completely abolishes urinary excretion of urate in man reducing it to $0.5 \%$ of filtered urate even in the face of a purine load. This leads to a sharp rise in plasma urate which limits the use of the drug therapeutically. Studies in some other species showing net secretion of urate--for example, the Dalmatian dog, suggested that PZA acted purely by blocking tubular secretion of urate, and this was assumed to apply to man with the additional assumption that secretion of urate in the nephron occurred at a site distal to the reabsorptive site.

More recent data have necessitated further modification of this "three component" filtrationreabsorption-secretion model of urate handling. There is increasing evidence to suggest that at least some of the tubular reabsorption occurs at a site distal to the secretory site. ${ }^{4}$ Thus, studies of the effect of PZA in patients with tubular disease and an. abnormally high excretion of urate (see below) showed, surprisingly, that the drug all but abolished urate excretion in these individuals, just as in normal subjects. PZA was also found to blunt considerably the response to uricosuric agents, such as probenecid, in healthy individuals. These observations are most plausibly explained by supposing that more urate is secreted than is suggested by suppression with PZA alone, and that some of this secreted urate also is reaborbed more distally in the nephron. The data suggest further that the uricosuric drugs, or tubular disease, affect this distal reabsorptive site predominantly.

A further doubt has been thrown on the significance of estimates of the tubular secretion of urate using PZA by the observation that, in primates, at high plasma drug concentrations (>100 $\mu \mathrm{g} / \mathrm{ml})$ PZA is uricosuric, apparently decreasing reabsorption, rather than exerting its predominant effect of decreasing secretion at lower concentrations. In this respect, PZA resembles a number of other drugs affecting urate excretion (see below).

The present picture of urate handling in the nephron is summarised in Fig. 2. Approximately $100 \%$ of plasma urate is filtered, and almost all of it is reabsorbed at a proximal site. Secretion then 
occurs, equivalent to about $50 \%$ of the filtered urate, and $80 \%$ of this is reabsorbed at a more distal site, probably still within the proximal tubule. Thus, an amount of urate is finally excreted which approximates to only $10 \%$ of the filtered load.

A large number of factors affect the renal handling of urate. Displacement of urate from albumin is no longer thought to be of importance, but a number of physiological and pathological agents are capable of reducing urate excretion, and hence causing a rise in plasma urate (Fig. 3). Any of these, therefore, may lead to an acute attack of gout in a susceptible individual whose plasma urate concentration is already marginally raised. Several of these deserve comment. The best known of the physiological substances are organic acids such as lactate which interfere with tubular handling of urate; their overproduction may explain in part the hyperuricaemia associated with alcohol intake, with status epilepticus and with starvation. Diuretics cause plasma volume contraction, and fractional urate reabsorption increases under these circumstances, as does $\mathrm{Na}^{+}$and $\mathrm{HCO}_{3}{ }^{-}$reabsorption. A number of drugs which increase the excretion of urate at high doses decrease the excretion at low doses. All uricosuric agents so far studied exhibit this biphasic response (except benzbromarone), but the agent which is best known for this property is aspirin, because its effect changes from reduced excretion to increased excretion of urate within its therepeutic range. For most other agents except PZA the change occurs well below the usual dose, so that retention of urate is not usually observed. It can be postulated that this biphasic action is the result of differential

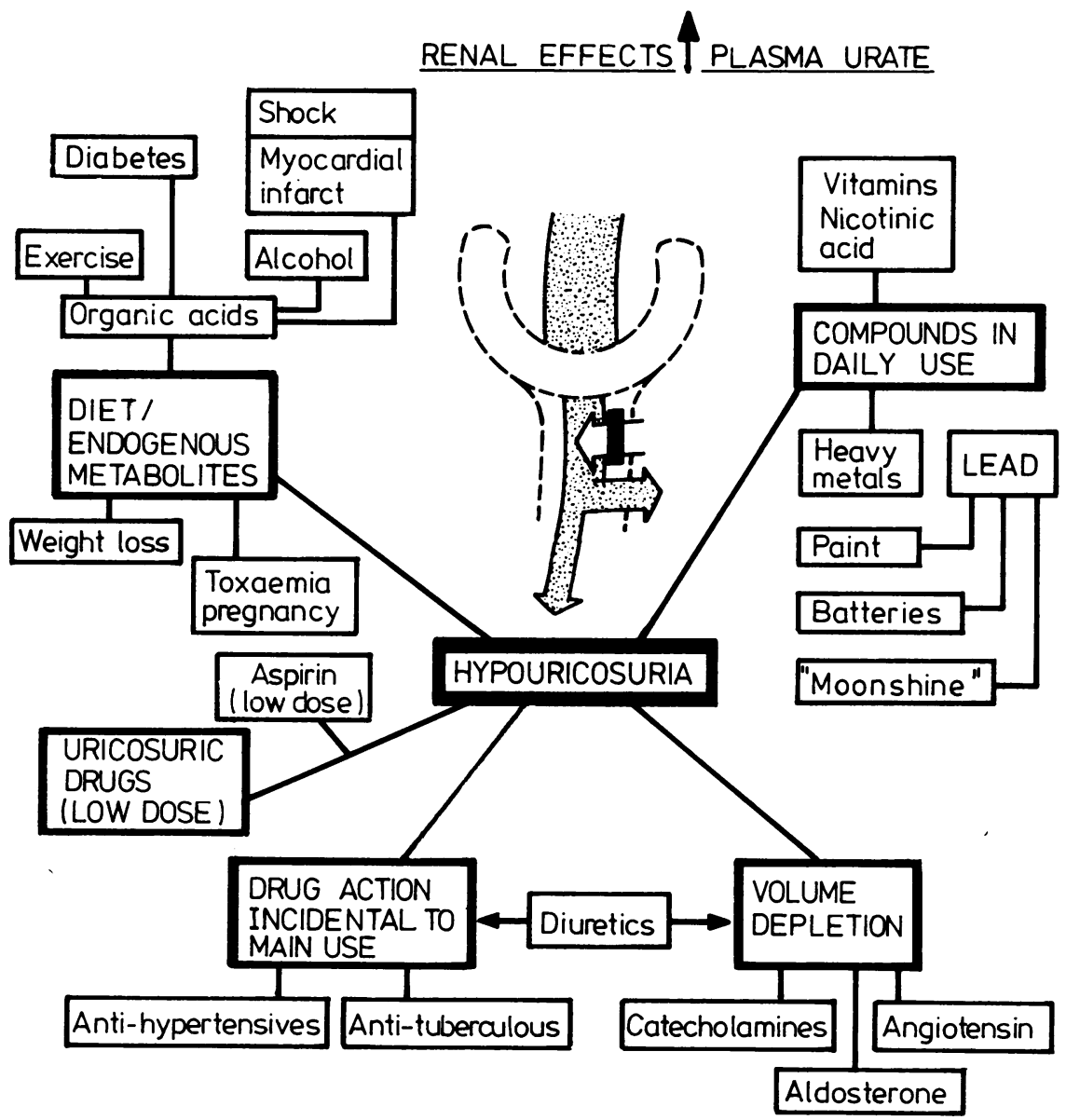

Fig. 3 A summary of factors tending to diminish net urate excretion so that a rise in plasma urate concentration follows. 
effects on secretion and postsecretory reabsorption of urate. Benzbromarone appears to be unique in that, unlike the other agents, it is not itself carried by the proximal tubular organic acid transport system, and therefore does not itself compete directly with the urate for whatever carrier is present. Chronic lead intoxication leads to a decrease in urate excretion by an undetermined mechanism; ${ }^{5}$ renal failure is a prominent and early manifestation, but arthritis ("saturnine" gout) is usually mild.

Those circumstances which lead to an increase of urate excretion and a fall of plasma urate are shown in Fig. 4. The most important of these are the group of uricosuric drugs. On occasion the excretion of urate following their administration may be so great as to lead to acute renal failure, through precipitation of urate in the tubules as was observed in some patients treated with the uricosuric diuretic tienilic acid (Ticrynafen). ${ }^{6}$ Circulatory volume expansion tends to increase urate excretion, and such expansion -for example, brought about by the inappropriate secretion of $\mathrm{ADH}$, may explain some of the hypouricaemias seen occasionally in patients with malignant disease. Massive doses of vitamin $\mathrm{C}$ are uricosuric and may lead to renal colic. Other agents not $\vec{\circ}$ normally thought of as affecting urate excretion also lead to considerable uricosuria, including most radiocontrast media, warfarin and corticosteroids.

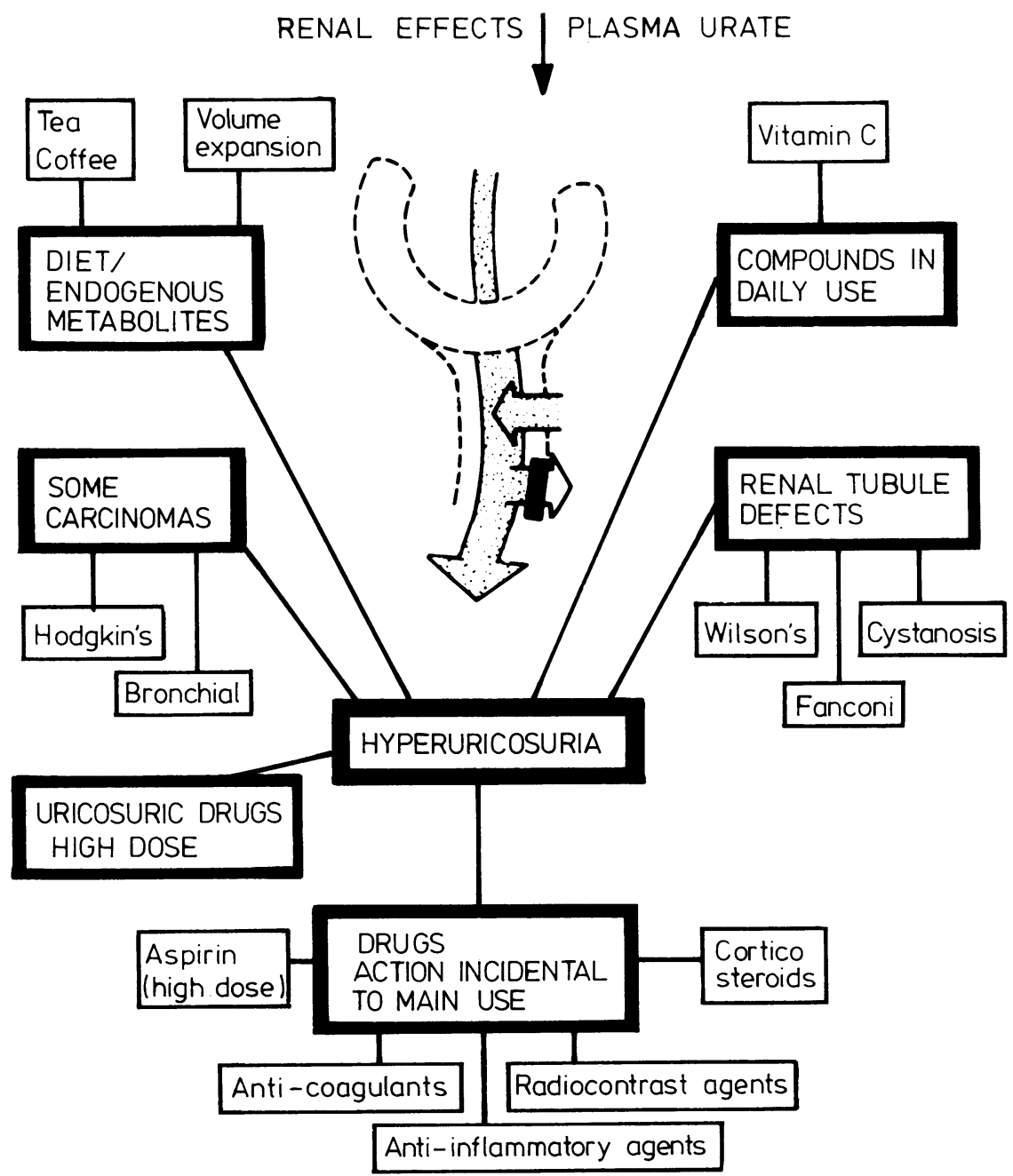

Fig. 4 A summary of factors tending to increase net urate excretion so that a fall in plasma urate concentration follows. 


\section{Uric acid in renal diseases}

T UBULAR DISEASE

It is worth mentioning first the rare abnormalities of renal tubular urate handling. Several families have been described with an isolated defect of tubular reabsorption of urate, in whom the clearance of urate may exceed the glomerular filtration rate, thus producing low plasma concentrations. These individuals are usually symptomless, and some are detected by routine estimation of plasma urate by multichannel analysers. PZA administration does not reduce the urate excretion in these individuals, and there are probably three different types of lesion distinguished, one affecting reabsorption throughout the whole nephron, the others only affecting pre- or postsecretory reabsorption.

Defects in urate reabsorption also occur in patients with generalised disease of the proximal tubule (the Fanconi syndrome) in whom abnormal urinary losses of glucose, amino acids, bicarbonate or phosphate may occur as a result of inherited or acquired tubular lesions-for example, cystinosis, Wilson's disease, galactosaemia. ${ }^{8}$ In such conditions attention is usually directed to those aspects of the syndrome which cause symptoms such as the acidosis or rickets arising from bicarbonate or phosphate wasting respectively, but these patients also have a very high clearance of urate and a very low plasma urate concentration.

Rather few studies of these lesions have been made, but PZA supresses the urinary excretion of urate, with the inference that the defect is principally one in postsecretory reabsorption.

\section{RENAL FAILURE}

The major problem in renal disease, however, is the renal handling of urate in patients with mild or severe uraemia. Obviously, humans and other primates are at risk of joining birds, snakes and other reptiles in developing widespread fatal deposition of uric acid crystals during uraemia. Fortunately, this does not happen and even clinical gout apparently secondary to chronic renal failure is very rare, being noted in only 17 of 1600 patients with uraemiz in one series. ${ }^{9}$

In uraemia, although the plasma concentrations of urea and creatinine may increase thirty to fortyfold, that of urate does not. Up to about twice the normal plasma concentration of creatinine (-that is, down to about half the normal glomerular filtration rate) the plasma concentration of uric acid rises approximately in parallel (Fig. 5) At this point the rise in the concentration of urate in the plasma flattens off despite a further fall in the glomerular filtration rate, so that the concentration of urate in

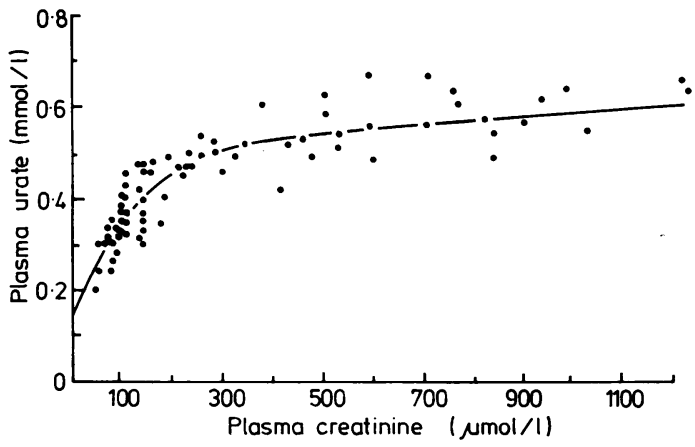

Fig. 5 The relation between plasma creatinine and plasma urate at various degrees of renal failure.

the plasma is still, on average, only about twice normal even in individuals with terminal uraemia. ${ }^{10}$

As renal failure progresses the urinary excretion of urate falls steadily, but much more slowly than would be predicted from the fall in the glomerular filtration rate, so that in terminal uraemia it is only one-half or one-third normal.11 Evidently the net tubular reabsorption of urate must fall from the normal value of about $90 \%$ in health, to as little as $20 \%$ in terminal uraemia. Correspondingly, the excreted fraction increases from $10 \%$ to as much as $80 \%$ of that filtered. ${ }^{12}$ How this adaptation occurs is not clear, and is unlikely to be settled until there is more accurate information on the tubular handling of urate in health. Some compounds retained in uraemia are uricosuric-for example, hippurates, and these may be the factors responsible for this aspect of adaptation to the uraemic state.

Even so, the reduction in total excretion of urate suggests either that urate production is reduced or that some extrarenal excretion increases as renal function declines. Although there has been some controversy, and data are not extensive, the consensus from studies using ${ }^{14} \mathrm{C}$-urate is that urate production in uraemia is normal. Also, it is known that in health some urate, probably one-third, but perhaps as little as one-tenth of the total production, is excreted into the gut and degraded there by uricase-bearing bacteria. This almost certainly increases in uraemia, although direct information on this point is limited to the study of two patients.

\section{ROLE OF THE KIDNEY IN THE GENESIS OF} GOUT

One hundred and thirty-two years ago, Garrod speculated that the cause of gout might be "a loss of power ... in the uric acid excretory function of the kidney." Before we can examine the suggestion that gout-or at least some gouty patients-suffer from a disorder of urate excretion, we must examine 
briefly the origins of uric acid. The body pool of urate, and hence the plasma urate concentration, is the result of a balance between production, ingestion, and excretion (Fig. 6). Ingested nucleotides and purines are largely degraded by gut and liver enzymes, whilst endogenous purine synthesis matches that incorporated into tissue nucleotides RNA and DNA. The synthesis of each molecule of purine requires the consumption of six molecules of ATP, but the bulk of the purine bases released from nucleotide degradation are "salvaged" for new nucleotide synthesis by the expenditure of a single molecule of ATP, principally through the agency of the enzyme hypoxanthine-guanine phosphoribosyl transferase (HGPRT, EC 2.4.2.8 ${ }^{13}$ ). The free nucleotides are powerful inhibitors of de novo purine synthesis, and thus this enzyme functions as part of an inhibitory pathway limiting and regulating purine synthesis, restricting the availability of pyrophosphate-ribose phosphate (PP-ribose-P) in the process.

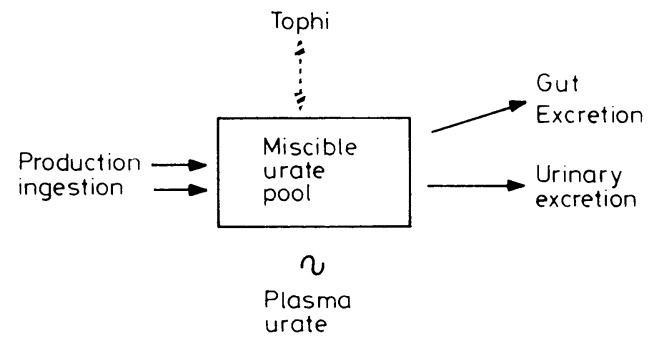

Fig. 6 Factors important in the production and disposal of urate in the body.

The possible causes of increased plasma urate concentration are (i) overproduction of endogenous purine, (ii) increased intake of exogenous dietary purine, (iii) decreased excretion of purine.

Purine production can be measured using the incorporation of radiolabelled precursors, but such methods are not suitable for use in routine clinics. A simpler way of assessing the de novo production of purines is to place the patient on a purine-free diet for five to seven days and measure the urinary excretion of urate, since by this time purine excretion will equal endogenous production. In this way less than $10 \%$ of patients with gout have been found to excrete an abnormally large amount of urate ( $>4$ $\mathrm{mmol} /$ day). In most of these the overproduction is modest, but in a few individuals great quantities of urate are produced-up to 5 or even 10 times normal. In some of these patients abnormalities of purine enzymes can be discerned, the best-known of which is the Lesch-Nyhan syndrome due to the absence or partial absence of the regulatory salvage enzyme HGPRT. ${ }^{13}$ Most of the hemizygotes for this deficiency are mentally defective and exhibit athetosis and a remarkable, bizarre tendency to selfmutilation. Others (and all heterozygotes) are much less affected, and may present as otherwise normal gouty overproducers of urate. Another enzyme. PP-ribose-P synthetase (EC 2.7.6.1.), may be present in a mutant form which has increased activity, and which consequently accelerates purine synthesis. This condition, like the Lesch-Nyhan syndrome, is inherited as a sex-linked recessive trait since the DNA coding for the enzyme is carried on the $X$ chromosome.

Purine ingestion has been known for millenia to be high in subjects with gout, as the popular stereotype of the gouty subject shows. Gout is a disorder of affluent societies with diets rich in purines; during times of hardship uric acid concentrations fall and clinical gout almost vanishes. These observations, and the extensive data on the increase in urinary purine and plasma urate concentrations in normal and gouty subjects on increasing purine intake, show the importance of dietary purine in precipitating clinical gout. The nature of the ingested purine may be more important than is realised, as some forms of purine-for example, from RNA, are absorbed and catabolise $\phi$ more readily than others.

However, neither overproduction (unless sudde and massive) nor overingestion are likely to raise the concentration of urate in the plasma, if the renal response is normal. When normal subjects have their urate production reduced by the administration of allopurinol, or raised by ingestion of yeast, which is rich in RNA, then the plasma urate rises little with increased intake, the increase in the excretion of urate being dramatic when the rise in plasma urate is modest (Fig. 7). When the same studies are undertaken on gouty subjects, an immediate difference is apparent (Fig. 7), some of which comes from the inclusion of normo- and hyperexcretors of urate. The striking difference is that for any plasma urate concentration, the gouty patients' excretion of urate was consistently less than in the normal subjects. Looked at another way, these data demonstrate that to generate the same excretion of urate, the gouty patient must "run" a higher plasma urate concentration.

The nature of this defect is not yet clear, since so many early studies must be re-evaluated in the light of recent knowledge of how complex the renal tubular handling of urate is. However, some recent evidence from studies using PZA or benzbromarone suggests that gouty patients underexcrete urate because of a defect in tubular secretion. ${ }^{4}$

These findings suggest that, in the majority of 


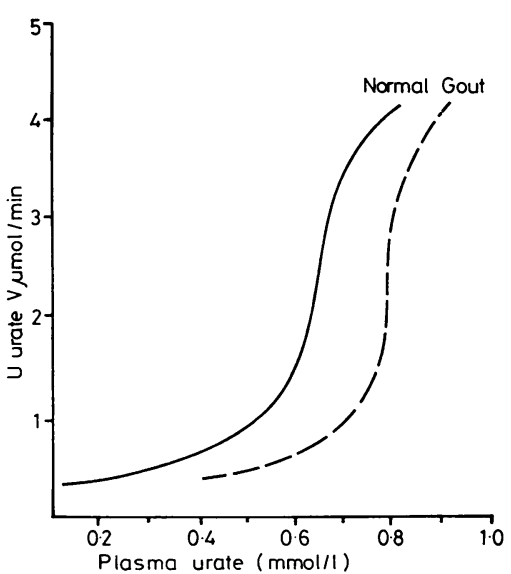

Fig. 7 The relation between plasma urate concentration and urate excretion in normal individuals and gouty subjects, when plasma urate varies spontaneously or in response to treatment (modified from Wyngaarden and Kelley, 1972). ${ }^{25}$

gouty patients, a combination of events is needed to produce hyperuricaemia and the clinical syndrome of gout. These are overproduction of urate, usually modest and probably genetically determined, or a large intake of readily-absorbed purines, together with a defect of the kidney which cannot respond to a purine load without an abnormal rise in plasma urate concentration. Thus, renal hypoexcretion of uric acid can be considered one of the major risk factors determining the appearance of clinical gout.

This raises the unsolved question of why clinical gout is not commoner than it is in patients with raised plasma urate as a result of renal failure. Two possible explanations have been advanced, neither of which is convincing. One suggests that urate is more soluble in uraemic than in normal plasma, the other that the inflammatory response normally evoked by urate crystal deposition is muted by the immunosuppression associated with uraemia.

The kidney also seems to be the site of the difference between men and women in their liability to gout. The female kidney excretes a higher proportion $(10-12 \%)$ of filtered urate than the male kidney $(8-10 \%)$, which gives males their higher plasma urate and makes them more vulnerable to overproduction or overingestion. ${ }^{3}$ This difference is not seen in postmenopausal women who thus become more liable to gout.

GOUTY NEPHROPATHY AND ACUTE

HYPERURICAEMIC RENAL FAILURE ${ }^{14}$

Urinary abnormalities such as mild proteinuria and abnormalities of urinary sediment are common in gouty patients, but although renal failure was common and one of the principal causes of death from gout until 20-30 yr ago, it is now rare. ${ }^{15} 16$ The cause of renal faiiure in gout has been much debated. Some suggest that all the renal damage follows the associated vascular disease of gout, ${ }^{15}$ while others see a central role for the deposition of uric acid or urate crystals within the kidney, which is the only feature specifically associated with gout. ${ }^{14}$

The reasons for the decline in the mortality from renal failure in gout are now impossible to determine, but it is tempting to relate them to the decreasing frequency of the disease associated with moderation of purine intake and with the more effective treatment of the disease, first with uricosuric agents and then with allopurinol. Today, gross deforming tophaceous gout is a rarity, and associated hypertension will normally receive effective treatment.

The nature of "gouty nephropathy"-or even its existence as such-has been the subject of much debate. On the clinical side there is no doubt that some patients with classical gout-men of middle age with a high purine intake in the form of food or beer-suffer a gradual decline in renal function which can be arrested by control of their plasma urate concentrations using allopurinol. However, a number of such patients are also hypertensive, and in many it is difficult to assess the relative importance of treating hyperuricaemia and hypertension. Equally, it is clear that this type of patient is now less common, and the decline in numbers of this formerly common group has brought another group of gouty patients with renal failure into prominence. These are young patients aged between 20-40 yr with precocious onset of gout in childhood, or early adult life, in whom renal failure is an early feature, men and women being affected equally. A family history of gout is common in this group, and in some of these families hypertension is absent initially, so it seems clear that this cannot be the feature causing the renal failure. Renal biopsies show an interstitial nephropathy with tubular atrophy and glomerulosclerosis, but urate crystals are rare. ${ }^{17}$

Experimentally, it has been shown that crystal nephropathies due to various purines, including xanthine and uric acid, can cause interstitial scarring as a long-term result. ${ }^{18}$ At this point the question of sodium urate and uric acid must be raised. In the interstitium of the kidney the ambient $\mathrm{pH}$ is that of plasma, and the predominant species of purine is the needle-shaped sodium urate. Within the tubules, however, the $\mathrm{pH}$ may be 5.0 or less and the predominant species will be amorphous uric acid, and there is no doubt that the predominant lesion in patients with acute overproduction of urate due to 
treatment with cytotoxic drugs (discussed below) is intratubular deposition of uric acid. The origin of the interstitial amorphous sodium urate in chronic gout nephropathy is controversial. Some see it as the result of the ambient plasma urate concentration, others (including ourselves) as the result of erosion of uric acid crystals out of the tubules with subsequent transformation to sodium urate. Both mechanisms may be operative; in any case, both uric acid and sodium urate are capable of generating inflammation with secondary scarring.

This argument is not merely academic, because if the primary abnormality is the deposition of sodium urate interstitially, appropriate treatment is to lower the plasma urate concentration and the method of achieving this is unimportant. If, on the other hand, the initial event is the intratubular deposition of uric acid crystals, then the tubular concentration of uric acid will be seen as the crucial event, and uricosuric agents will be contraindicated. It seems best therefore in patients with gout and renal impairment to reduce urate production with allopurinol, which should be effective in either case. It must be remembered, however, that its active metabolite, oxipurinol, even in patients with normal kidney function, has a long half-life since, unlike its parent drug, it is reabsorbed in the tubules; and the dose of allopurinol may have to be reduced to as little as $100 \mathrm{mg}$ daily or even $100 \mathrm{mg}$ three times weekly in advanced renal failure, to minimise the risk of bone marrow depression.

In some patients, even control of both hypertension and plasma urate concentration fails to arrest a decline in renal function, and this again raises the question of exactly what determines the appearance, severity and progression of interstitial nephropathy in gouty patients. It seems likely that there are several groups which can be identified rather tentatively, some of which respond to available treatment. Even so, the central fact is that, today, chronic renal failure is rare in gout. Gout is the primary cause of renal failure in less than $1 \%$ of patients treated by regular dialysis or transplantation in Europe as a whole, or even in our own unit which has a particular interest in gouty nephropathy. ${ }^{19}$

Fortunately, the acute hyperuricaemic renal failure $^{20}$ which occurs most commonly when there is massive overproduction of purines as a result of tissue breakdown, also is becoming rare. The commonest circumstance is the treatment of leukaemia, lymphoma or myeloma, but rare instances after spontaneous remission or the treatment of solid tumours have been recorded. The appearance of renal failure can be averted in almost all cases by ensuring a high volume of alkaline urine by means of a high fluid intake coupled with a diuretic and sodium bicarbonate, and above all, by giving allopurinol to distribute the purine load over the three purines hypoxanthine, xanthine and uric acid, through its action in blocking xanthine oxidase. Whilst hypoxanthine is very soluble, xanthine is as insoluble as uric acid in neutral and acid urine but its solubility does not increase in alkaline urine. A few cases of xanthine stones or xanthine nephropathy have been reported in patients treated for malignancies with allopurinol, and also occasionally in other patients with gross overproduction of urate. such as the Lesch-Nyhan syndrome, treated similarly.

\section{URIC ACID STONES}

Urate is least soluble in the form of uric acid in acid urines (Fig. 1). An interesting observation is that many patients with gout, but otherwise normal renal function, pass urine with a low $\mathrm{pH}$ in the range $4 \cdot 8-5 \cdot 4$ throughout the 24 hours of the day. This contrasts with normal individuals, who, depending on their diet, show a $\mathrm{pH}$ above 6 at some time in the day as part of diurnal variation. A persistently low urine $\mathrm{pH}$ is also seen in some non-gouty uric acid stone-formers, and is associated with a defective tubular secretion of ammonium, the mechanism of which is obscure ${ }^{10}$ - the often-quoted idea that thi results from diversion of glutamate from ammoniun to urate production has been shown to be untenable on quantitative grounds. In young patients with gout stone formation often precedes the onset of the arthritis.

The importance of the acid urine in predisposing to the formation of urate stones is clear, as Fig. 1 shows. Obvious steps in treatment are: (i) to increase urine volume, (ii) to increase the $\mathrm{pH}$ of the urine, (iii) to reduce urinary urate excretion by allopurinol. In practice, the first two alone usually suffice ${ }^{21}$ but it is important to realise that sodium itself, given as sodium bicarbonate, actually decreases the solubility of urate in the urine, by a common ion effect. It is important, therefore, to ensure that the urine volume is greatly increased, since the solubility of urate in the urine increases as the square of the volume, again because of interaction with other ions.

An interesting observation is that gouty patients also have an increased tendency to form oxalate stones. This may be related to competition by excess uric acid for inhibitors of crystallisation in the urine, or to the action of uric acid crystals as nuclei for the crystallisation of the calcium oxalate; but an effect of purine metabolism on oxalate production is also possible. In passing we can note that in addition to uric acid and xanthine, an even more insoluble purine, namely 2,8 dihydroxyadenine, (2,8-DHA), the 6 -amino analogue of uric acid, may on rare 
occasions form radiolucent stones which are easily confused with uric acid chemically, although the appearance is distinctive; $;^{22}$ stones of 2,8-DHA are blue grey and crumbly, in contrast to the hard, yellowish urate stones. 2,8-DHA is excreted in individuals who are homozygotes for the deficiency of adenine phosphoribosyltransferase (APRT, EC 2.4.2.7), the companion purine salvage enzyme to HGPRT. This deficiency makes adenine available for oxidation by xanthine oxidase to $2,8-\mathrm{DHA}$, so that allopurinol should be effective treatment. Unlike HGPRT, APRT is not apparently important in the regulation of de novo purine synthesis, so these patients do not have gout. They may, however, present in renal failure, either because of urinary tract obstruction by the stones, or because of a diffuse interstitial nephritis associated with 2,8-DHA crystals in the tubules and interstitium. The latter observation supports the idea that intratubular urate is important in producing the interstitial nephritis of gout.

\section{SYMPTOMLESS HYPERURICAEMIA}

Since we now have, in allopurinol, a powerful tool for the reduction of plasma urate, it has been questioned whether allopurinol should be used when a raised plasma urate concentration occurs without apparent harmful effects. The first such condition is symptomless hyperuricaemia usually, but not always, found in association with mild hypertension. Treatment of the latter with thiazide diuretics, of course, may further increase the plasma urate and may precipitate acute gout. A raised plasma urate concentration has been identified as a risk factor for vascular disease. ${ }^{6}$ A study of hypertensives with symptomless hyperuricaemia for up to four years failed to show any difference in creatinine clearance, ${ }^{23}$ but this does not exclude a longer term effect. If the plasma urate persistently exceeds $0.60 \mathrm{mmol} / \mathrm{l}$ the likelihood of an attack of clinical gout is very high, and prophylactic treatment is justified. At present no secure advice can be given for those with a plasma urate in the range $0.45-0.60 \mathrm{mmol} / \mathrm{l}$, except that dietary purines should be restricted.

The second such condition is uraemia from causes other than gouty nephropathy. In fatal cases urate crystals have been demonstrated in the renal medulla in alcohol-fixed material, and this has led to the suggestion that the decline in renal function might be accelerated by the raised plasma urate. ${ }^{24}$ The likelihood of this depends on whether the interstitial urate plays an important part in the pathogenesis of gouty nephropathy, and whether it originates from ambient plasma urate or from tubular uric acid crystals. Allopurinol has not been tried in non-gouty uraemics, and for the moment, in view of its side effects and the accumulation of its metabolite oxipurinol in renal failure, such treatment seems too speculative to recommend.

\section{TRANSPLANTATION AND LEUKAEMIA}

Finally, the dangers of combined treatment with allopurinol and purine analogues needs to be mentioned. Both azathioprine and its metabolite, 6-mercaptopurine are oxidised to thiouric acid by xanthine oxidase, and allopurinol prolongs the actions of the drugs indefinitely. Even more interestingly, in cyclophosphamide-treated patients leucopenia is more frequent in patients also given allopurinol.

\section{References}

${ }^{1}$ Roch-Ramel F. Renal excretion of uric acid in mammals. Clin Nephrol 1979;12:1-6.

${ }^{2}$ Steele TH, Rieselbach RE. The renal handling of urate and other organic anions. In: Brenner BM, Rector FC, eds. The kidney Vol 2. Philadelphia: WB Saunders, 1976: 442-67.

${ }^{3}$ Wolfson WQ, Hunt HD, Levine R, et al. The transport and excretion of uric acid in man. J Clin Endocrinol 1949; 9:749-66.

${ }^{4}$ Levinson DJ, Sorensen LB. Renal handling of uric acid in normal and gouty subjects: evidence for a 4 component system. Ann Rheum Dis 1980;39:173-9.

${ }^{5}$ Emmerson BT. Chronic lead nephropathy. Kidney Int $1973 ; 4: 1-5$.

${ }^{6}$ Anonymous. Diuretics, hyperuricaemia and tienilic acid. Lancet 1980;ii:681-2.

${ }^{7}$ de Vries A, Sperling O. Inborn hypouricemia due to isolated tubular defect. Biomedicine 1979;30:75-80.

${ }^{8}$ Wilson DM, Goldstein NP. Renal urate excretion in patients with Wilson's disease. Kidney Int 1973;4: 331-6.

${ }^{9}$ Richet G, Mignon F, Ardaillou R. Goutte secondaire des néphropathies chroniques. Presse Médicale 1965;73: 633-8.

${ }^{10}$ Hatfield PJ, Simmonds HA. Uric acid and the kidney: Current concepts. Guy's Hospital Reports 1974;123: 271-97.

11 Emmerson BT, Row PG. An evaluation of the pathogenesis of the gouty kidney. Kidnev Int 1975;8:65-71.

12 Danovitch GM, Weinberger J, Berlyne GM. Uric acid in advanced renal failure. Clin Sci 1967;43:331-41.

${ }^{13}$ Seegmiller JE. Human aberrations of purine metabolism and their significance for rheumatology. Ann Rheum Dis 1980;39:103-17.

${ }^{14}$ Cameron JS, Simmonds HA. Gout and crystal related nephropathy. Contrib Nephrol 1979;16:147-53.

${ }^{15}$ Berger L, Yü TF. Renal function in gout. IV. An analysis of 524 gouty subjects including long-term follow-up studies. Am J Med 1975;59:605-13.

${ }^{16}$ Gibson T, Simmonds HA, Potter CF, Jeyarajah N, Highton J. Gout and renal failure. Eur J Rheum Inflam $1978 ; 1: 79-85$.

17 Simmonds HA, Warren DJ, Cameron JS, Potter CF, Farebrother DA. Familial gout and renal failure in young women. Clin Nephrol 1980;14:176-82.

18 Farebrother DA, Hatfield P, Simmonds HA, Cameron JS, Jones AS, Cadenhead A. Experimental crystal nephropathy. One year study in the pig. Clin Nephrol 1973; 4:243-50. 
19 Simmonds HA, Cameron JS, Potter CF, Warren D, Gibson T, Farebrother D. Renal failure in young subjects with familial gout. In: Rapado A, Watts RWE, de Bruyn CHMM, eds. Purine metabolism in man III Part A. New York: Plenum Press, 1980:15-20.

20 Robinson RR, Yarger WE. Acute uric acid nephropathy in man. Arch Intern Med 1977;137:839-40.

21 de Vries A, Sperling O. Recent data on uric acid lithiasis. Adv Nephrol 1974;3:89-116.

${ }^{22}$ Simmonds HA. 2,8-dihydroxyadeninuria-or when is a uric acid stone not a uric acid stone? Clin Nephrol 1979; 12:196-7.

${ }^{23}$ Rosenfeld JB. Effect of long-term allopurinol administration in normotensive and hypertensive hyperuricemic subjects. In: Sperling O, de Vries A, Wyngaarden JB, eds. Purine metabolism in man. New York: Plenum Press, 1976:581.
${ }^{24}$ Verger D, Leroux-Robert C, Gauter P, Richet G. Les tophus goutteux de la medullaire rénale des urémiques chroniques. Nephron 1967;4:356-70.

${ }^{25}$ Wyngaarden JB, Kelley WN. In: Stanbury JB, Wyngaarden JB, Fredrickson DS, eds. Metabolic basis of inherited disease 3rd ed. New York: McGraw-Hill, 1972: 889.

NB A detailed account of all aspects of gout and the kidney is to be found in Wyngaarden JB, Kelley WN. Gout and hypruricemia. New York: Grune \& Stratton, 1976.

Requests for reprints to: Prof JS Cameron, Clinical Science Laboratories, 17th Floor Guy's Tower, Guy's Hospital, London SE1 9RT, England. 